Draft Version SePtember 12, 2018

Preprint typeset using LATEX style emulateapj v. 12/16/11

\title{
DETECTION OF GEV $\gamma$-RAY EMISSION IN THE DIRECTION OF HESS J1731-347 WITH FERMI-LAT
}

\author{
Xiao-Lei Guo ${ }^{1,2}$, Yu-Liang Xin ${ }^{1,3}$, Neng-Hui Liao ${ }^{1,2}$, Qiang $_{\text {Uan }}{ }^{1,2}$, Wei-Hong GaO $^{4,5}$, Yi-Zhong Fan $^{1,2}$ \\ ${ }^{1}$ Key laboratory of Dark Matter and Space Astronomy, Purple Mountain Observatory, Chinese Academy of Sciences, Nanjing 210008, China \\ ${ }^{2}$ School of Astronomy and Space Science, University of Science and Technology of China, Hefei 230026, Anhui, China \\ ${ }^{3}$ University of Chinese Academy of Sciences, Yuquan Road 19, Beijing, 100049, China \\ ${ }^{4}$ Department of Physics and Institute of Theoretical Physics, Nanjing Normal University, Nanjing 210046, China and \\ ${ }^{5}$ INAF-Osservatorio Astronomico di Brera, via E. Bianchi 46, 23807 Merate, Italy \\ ylxin@pmo.ac.cn (YLX); yuanq@pmo.ac.cn (QY); gaoweihong@njnu.edu.cn (WHG)
}

Draft version September 12, 2018

\begin{abstract}
We report the detection of $\mathrm{GeV} \gamma$-ray emission from supernova remnant HESS J1731-347 using 9 years of Fermi Large Area Telescope data. We find a slightly extended GeV source in the direction of HESS J1731347. The spectrum above $1 \mathrm{GeV}$ can be fitted by a power-law with an index of $\Gamma=1.77 \pm 0.14$, and the $\mathrm{GeV}$ spectrum connects smoothly with the TeV spectrum of HESS J1731-347. Either a hadronic-leptonic or a pure leptonic model can fit the multi-wavelength spectral energy distribution of the source. However, the hard $\mathrm{GeV} \gamma$-ray spectrum is more naturally produced in a leptonic (inverse Compton scattering) scenario, under the framework of diffusive shock acceleration. We also searched for the $\mathrm{GeV} \gamma$-ray emission from the nearby $\mathrm{TeV}$ source HESS J1729-345. No significant GeV $\gamma$-ray emission is found, and upper limits are derived.

Subject headings: gamma rays: general - gamma rays: ISM - ISM: individual objects (HESS J1731-347) ISM: supernova remnants - radiation mechanisms: non-thermal
\end{abstract}

\section{INTRODUCTION}

It is widely believed that supernova remnants (SNRs) are the main accelerators of Galactic cosmic rays (CRs) with energies up to the knee. This is supported by the non-thermal X-ray emission detected in many SNRs, which indicates the acceleration of electrons to hundreds of $\mathrm{TeV}$ energies (e.g., Koyama et al. 1995). The GeV and/or TeV $\gamma$-rays have also been detected in some SNRs, for example, RCW 86 (Aharonian et al. 2009; Yuan et al. 2014); Cas A (Albert et al. 2007a; Abdo et al. 2010a); CTB 37B (Aharonian et al. 2008a; Xin et al. 2016); Puppis A (Hewitt et al. 2012; Xin et al. 2017); IC 443 (Albert et al. 2007b; Acciari et al. 2009; Ackermann et al. 2013); W44 (Abdo et al. 2010b; Ackermann et al. 2013). Gamma-rays can be produced by the decay of neutral pions due to the inelastic $p p$ collisions (the hadronic process), the Inverse Compton Scattering (ICS) or bremsstrahlung process of relativistic electrons (the leptonic process). For some SNRs interacting with dense molecular clouds, the evidence for acceleration of nuclei has been suggested by $\mathrm{GeV} / \mathrm{TeV} \gamma$-ray observations (Li \& Chen 2010, 2012; Ackermann et al. 2013).

HESS J1731-347 (G353.6-0.7) was first observed as an unidentified very-high-energy (VHE; >100 GeV) $\gamma$-ray source by the High Energy Stereoscopic System (HESS) (Aharonian et al. 2008b). Tian et al. (2008) discovered the radio and X-ray counterparts of HESS J1731-347 and identified it as a shell-type SNR. Abramowski et al. (2011) carried out an additional $\gamma$-ray observation with HESS and detected its shell-type morphology. Together with RX J1713.7-3946 (Aharonian et al. 2004, 2006, 2007a), RX J0852.0-4622 (Aharonian et al. 2005, 2007b), RCW 86 (Aharonian et al. 2009; Abramowski et al. 2016) and SN 1006 (Acero et al. 2010), HESS J1731-347 becomes one of five firmly identified TeV shell-type SNRs (Rieger et al. 2013).

The distance of HESS J1731-347 is under debated. Tian et al. (2008) argued that HESS J1731-347 locates at $\sim 3.2 \mathrm{kpc}$ if it is associated with the nearby HII region G353.42-0.37. By comparing the absorption column density derived from the X-ray observation and that obtained from ${ }^{12} \mathrm{CO}$ and HI observations, Abramowski et al. (2011) set 3.2 $\mathrm{kpc}$ as a lower limit of its distance, which is reinforced by Doroshenko et al. (2017). In addition, Fukuda et al. (2014) suggested that HESS J1731-347 is correlated with the interstellar proton cavity at a velocity range from $-90 \mathrm{~km} \mathrm{~s}^{-1}$ to $-75 \mathrm{~km} \mathrm{~s}^{-1}$, indicating a distance of $5.2-6.1 \mathrm{kpc}$. However, no significant emission from dense molecular gas traced by CS(1-0) line coincides with HESS J1731-347 at that distance (Maxted et al. 2017). Due to uncertainties of the distance and other parameters, the age of HESS J1731-347 is estimated to be in a wide range of $2-27 \mathrm{kyr}$ (Tian et al. 2008; Abramowski et al. 2011; Fukuda et al. 2014; Acero et al. 2015b).

HESS J1731-347 and its sub-regions were detected in the X-ray band by ROSAT, XMM-Newton and Suzaku, with an Xray morphology consistent with the radio shell (Tian et al. 2008, 2010; Abramowski et al. 2011; Bamba et al. 2012; Doroshenko et al. 2017). The X-ray emission from the complete SNR and its sub-regions are found to be nonthermal. The X-ray spectral index is 2.66 for the entire SNR (Doroshenko et al. 2017), and is somewhat harder $(\Gamma=2.28)$ for the north-east region (Abramowski et al. 2011). A compact object, XMMS J173203-344518, located near the geometrical center of the remnant, was detected by XMMNewton, which was considered to be the central compact object (CCO) associated with HESS J1731-347 (Tian et al. 2010; Halpern \& Gotthelf 2010; Abramowski et al. 2011).

Yang et al. (2014) and Acero et al. (2015b) searched for the GeV $\gamma$-ray emission from HESS J1731-347 with the Fermi Large Area Telescope (Fermi-LAT; Atwood et al. 2009) data. No significant signal was detected and only the upper limits were given. Furthermore, no candidate source in the third Fermi-LAT source catalog (3FGL; Acero et al. 2015a) is found to be associated with HESS J1731-347. 
In this paper, we revisit the $\mathrm{GeV} \gamma$-ray emission in the direction of HESS J1731-347, with 9 year Pass 8 data recorded by Fermi-LAT. A statistically significant excess which is positionally consistent with HESS J1731-347 is found. In Section 2, we present the data analysis and results, including the spatial and spectral analysis. Based on the multi-wavelength observations of HESS J1731-347, we model the non-thermal radiation of it in Section 3. The conclusion of this work is presented in Section 4.

\section{DATA ANALYSIS}

\subsection{Data Reduction}

We select the latest Pass 8 version of the FermiLAT data with "Source" event class (evclass $=128$ \& evtype=3), recorded from August 4, 2008 (Mission Elapsed Time 239557418) to August 4, 2017 (Mission Elapsed Time $523497605)$. The region of interest (ROI) is chosen to be a $14^{\circ} \times 14^{\circ}$ box centered at HESS J1731-347. In order to have a good angular resolution, we adopt the events with energies between $1 \mathrm{GeV}$ and $300 \mathrm{GeV}$ in this analysis. In addition, the events whose zenith angles are larger than $90^{\circ}$ are excluded to reduce the contamination from the Earth Limb. The data are analyzed with the Fermi-LAT Science Tools v10r0p5 ${ }^{1}$, and the standard binned likelihood analysis method gtlike. The diffuse backgrounds used are gll_iem_v06.fits and iso_P8R2_SOURCE_V6_v06.txt, which can be found from the Fermi Science Support Center ${ }^{2}$. All sources listed in the 3FGL and the two diffuse backgrounds are included in the model. During the fitting procedure, the spectral parameters and the normalizations of sources within $5^{\circ}$ around HESS J1731-347, together with the normalizations of the two diffuse backgrounds, are left free.

\subsection{Results}

We create a $4^{\circ} \times 4^{\circ}$ TS (test statistic, which is essentially the logarithmic likelihood ratio between different models) map centered at HESS J1731-347 by slice the center of the box along each axis, after subtracting the 3FGL sources and the diffuse backgrounds. There are still excesses in this TS map, as marked out by green crosses. At the center of the TS map, a weak excess (labelled as Source T) is found to be spatially coincident with HESS J1731-347. It is noted that Newpts $C$ was also detected in Yang et al. (2014) with a TS value of about 20. We add Source T and the other six new sources, from $\mathrm{A}$ to $\mathrm{F}$, in the model as additional point sources with power-law (PL) spectra, and re-do the likelihood fitting. The positions of these new sources are optimized by the gtfindsrc tool. Best-fitting results of their coordinates and TS values are listed in Table 1 . The TS value of Source T is about 25.9, and its best-fitting position is R.A. $=262.902^{\circ}$, Dec. $=-34.775^{\circ}$ with $1 \sigma$ error circle of $0.022^{\circ}$. The residual TS map after subtracting the additional sources $\mathrm{A}$ to $\mathrm{F}$ is shown in the right panel Figure 1.

Figure 2 gives a $1.3^{\circ} \times 1.3^{\circ}$ zoom-in of Figure $1 \mathrm{~b}$ in order to better show the spatial distribution of the target source $\mathrm{T}$ and its relationship with HESS J1731-347. The GeV $\gamma$ ray emission overlaps with part of the VHE emission region shown by the contours (Abramowski et al. 2011). However, the GeV TS map does not fully overlap with the TeV image, which is possibly due to the large point spread function (PSF)

\footnotetext{
${ }^{1}$ http://fermi.gsfc.nasa.gov/ssc/data/analysis/software/

${ }^{2}$ http://fermi.gsfc.nasa.gov/ssc/data/access/lat/BackgroundModels.html
}

of Fermi-LAT and/or the fluctuation of the weak signal. Similar cases were also shown for SN 1006 (Xing et al. 2016) and HESS J1534-571 (Araya 2017).

TABLE 1

COORDINATES, TS valuES, AND ANGULAR SEPARATIONS FROM THE CENTER OF HESS J1731-347 OF THE NEWLY ADDED POINT SOURCES

\begin{tabular}{ccccc}
\hline \hline Name & $\begin{array}{c}\text { R.A. } \\
{[\mathrm{deg}]}\end{array}$ & $\begin{array}{c}\text { Dec. } \\
{[\mathrm{deg}]}\end{array}$ & TS & $\begin{array}{c}\Delta \theta \\
{[\mathrm{deg}]}\end{array}$ \\
\hline Source T & 262.902 & -34.775 & 25.9 & 0.093 \\
Newpts A & 264.048 & -34.370 & 130.8 & 0.936 \\
Newpts B & 262.629 & -33.882 & 29.3 & 0.929 \\
Newpts C & 262.280 & -35.051 & 59.8 & 0.670 \\
Newpts D & 260.867 & -33.704 & 59.5 & 2.062 \\
Newpts E & 262.266 & -36.233 & 34.9 & 1.598 \\
Newpts F & 265.161 & -34.500 & 26.5 & 1.786 \\
\hline \hline
\end{tabular}

\subsubsection{Spatial Extension}

Considering that HESS J1731-347 has an extended morphology in radio, $\mathrm{X}$-ray, and $\mathrm{TeV} \gamma$-ray bands, we carried out an extension test with different spatial models. We used a uniform disk centered at the best-fitting position of Source $\mathrm{T}$ with radius of $0.1^{\circ}, 0.15^{\circ}, 0.2^{\circ}$, and $0.25^{\circ}$, as well as the $\mathrm{TeV} \gamma$-ray image of HESS J1731-347, as spatial templates of Source T. The TS values for different spatial models are listed in Table 2. We found that a $0.15^{\circ}$ disk template gives the highest TS value, 33.9, which corresponds to a significance of $\sim 4.7 \sigma$ for five ( 2 for the coordinates, 1 for the radius, and 2 for the spectrum) degrees of freedom (dof). For the four adopted disk templates, the TS values do not differ much from each other. Compared with the point source hypothesis, the data favors slightly an extended morphology. Using the TeV $\gamma$-ray template, a TS value of 25 is found. These results are quite consistent with that of Condon et al. (2017), which used the data with different energy ranges and observation time-series. In the following analysis, we adopt the $0.15^{\circ}$ disk template for Source T.

We also try to search for $\gamma$-ray emission from the nearby TeV source HESS J1729-345. The TeV image of HESS J1729-345 is used as the spatial template. No significant GeV $\gamma$-ray emission from the direction of HESS J1729-345 is detected. The TS value of HESS J1729-345 is about 4, and its flux upper limits will be derived (see the next sub-section).

TABLE 2

TS VALUES OF SOURCE T WITH DIFFERENT SPATIAL MODELS

\begin{tabular}{ccc}
\hline \hline Spatial Model & TS & Degrees of Freedom \\
\hline Point Source & 25.9 & 4 \\
$0.1^{\circ}$ Uniform Disk & 32.1 & 5 \\
$0.15^{\circ}$ Uniform Disk & 33.9 & 5 \\
$0.2^{\circ}$ Uniform Disk & 32.4 & 5 \\
$0.25^{\circ}$ Uniform Disk & 32.4 & 5 \\
TeV Image & 25.0 & 2 \\
\hline \hline
\end{tabular}

Note. - The 4 dof for the point source model include 2 spatial and 2 spectral parameters. The uniform disk has 5 dof, 2 for coordinate, 2 for spectral parameters and 1 for radius. For the TeV image template model, only 2 dof of the spectral parameters are considered.

\subsubsection{Spectral Analysis}

For Source T, the global fit in the $1-300 \mathrm{GeV}$ energy range with a $0.15^{\circ}$ disk template gives a spectral index of $\Gamma=$ 


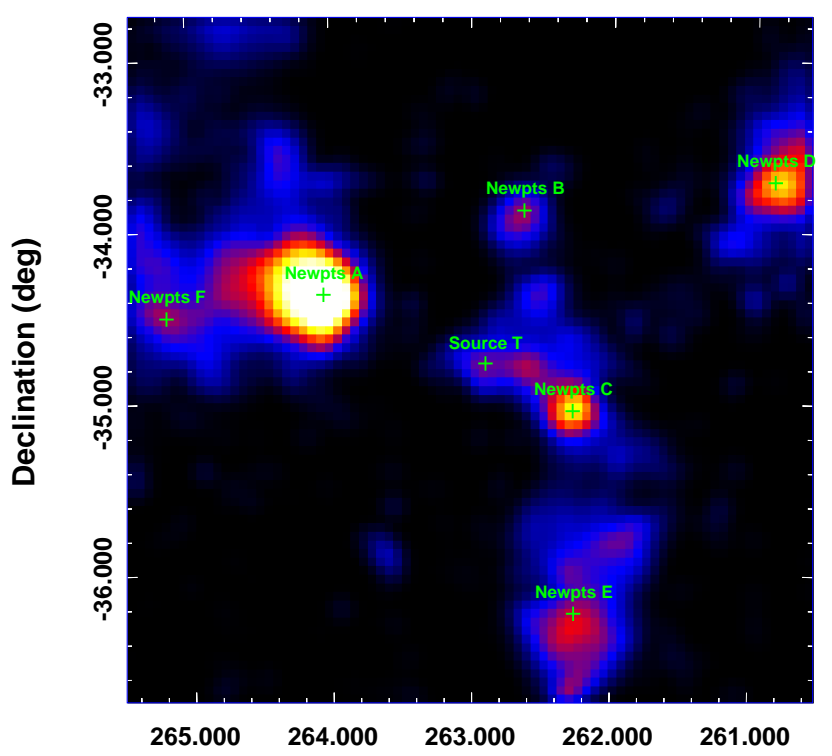

Right Ascension (deg)

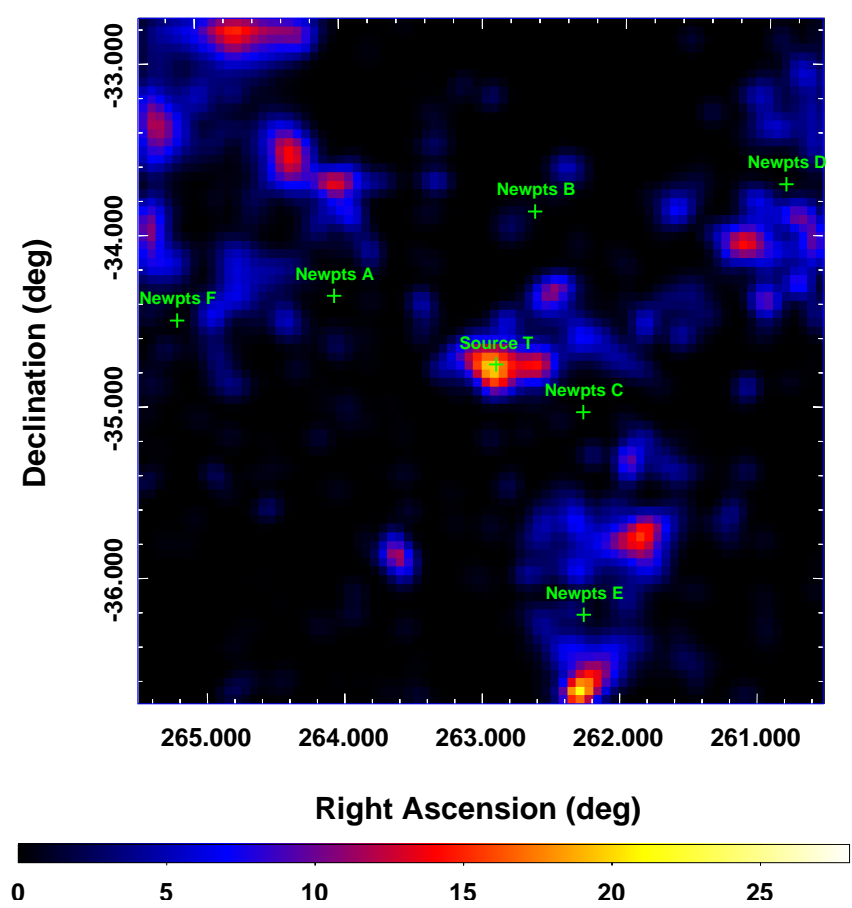

\begin{tabular}{llllllll}
\hline 0 & 10 & 20 & 30 & 40 & 50 & 60 & 70
\end{tabular}

FIG. 1.- TS maps of a $4^{\circ} \times 4^{\circ}$ region centered on the position of HESS J1731-347, for photons above 1 GeV. The left panel is the TS map with 3 FGL sources and the diffuse backgrounds subtracted, and the right panel is the TS map with the six additional new sources A to F (green crosses) subtracted. All maps are smoothed with a Gaussian kernel with $\sigma=0.1^{\circ}$.

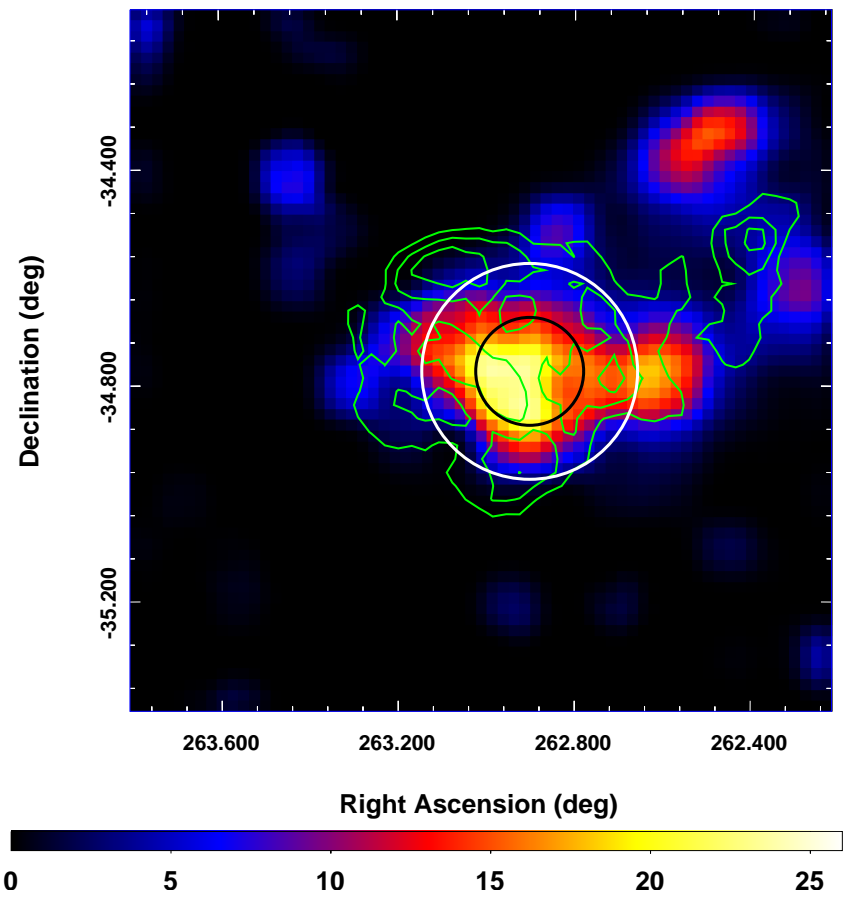

FIG. 2.- Zoom-in of the right panel of Figure 1, for a region of $1.3^{\circ} \times 1.3^{\circ}$ centered at HESS J1731-347, overlaid with the contours of the VHE image by HESS (Abramowski et al. 2011). The green contours to the west show the VHE image of HESS J1729-345. The radius of Gaussian smooth kernel is $\sigma=0.04^{\circ}$. The black and white circles mark the radii of $0.1^{\circ}$ and $0.2^{\circ}$ uniform disk, respectively.

$1.77 \pm 0.14$, and an integral photon flux of $(6.92 \pm 2.06) \times$ $10^{-10}$ photon $\mathrm{cm}^{-2} \mathrm{~s}^{-1}$ with statistical errors only. Assuming a distance of $3.2 \mathrm{kpc}$ (Tian et al. 2008; Nayana et al. 2017), the $\gamma$-ray luminosity between $1 \mathrm{GeV}$ and $300 \mathrm{GeV}$ is $1.26 \times$ $10^{34}\left(d / 3.2 \mathrm{kpc}^{2} \mathrm{erg} \mathrm{s}^{-1}\right.$.

The data is further divided into four energy bins with equal width in the logarithmic space to study is spectral energy distribution (SED). For each energy bin, we repeat the likelihood analysis, with only the normalizations of the sources within $5^{\circ}$ around Source $\mathrm{T}$ and the diffuse backgrounds in the model free. The spectral parameters of these sources are fixed to be the best-fitting values obtained in the global likelihood analysis. If the TS value of Source T is smaller than 4 in an energy bin, a 95\% confidence level upper limit is given. The results of the SED are shown in Figure 3. The GeV SED connects smoothly with the TeV spectrum of HESS J1731-347. The spatial coincidence and a smoothly connected $\gamma$-ray spectrum suggest that Source $\mathrm{T}$ is the $\mathrm{GeV}$ counterpart of HESS J1731-347.

The significance of HESS J1729-345 is not high enough, and we derive the flux upper limits in energy bins of $1-6.7$, $6.7-44.8$, and $44.8-300 \mathrm{GeV}$, which are shown in Figure 4.

\section{DISCUSSION}

The radio counterpart of HESS J131-347 was firstly identified by Tian et al. (2008). The integrated flux density was derived to be $2.2 \pm 0.9 \mathrm{Jy}$ at $1420 \mathrm{MHz}$, through extrapolating that of one half of the remnant at low Galactic latitudes to the total SNR. With the Giant Metrewave Radio Telescope (GMRT), Nayana et al. (2017) observed the complete shell of HESS J131-347 at $325 \mathrm{MHz}$, and obtained an integrated flux density of $1.84 \pm 0.15 \mathrm{Jy}$. In the following models, we use the result of Nayana et al. (2017) to constrain the model parameters. The X-ray flux of the full SNR given by Doroshenko et al. (2017) is also used.

We assume either a pure leptonic model or a hadronicleptonic hybrid one to fit the wide-band SED from radio to $\mathrm{TeV} \gamma$-rays. The spectrum of electrons or protons is assumed 


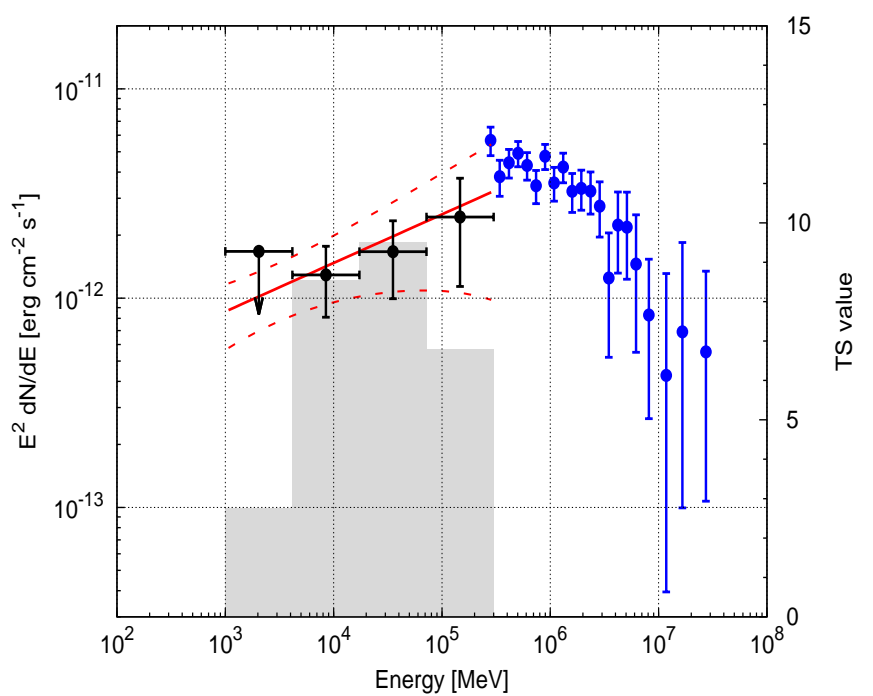

FIG. 3. - The Fermi-LAT SED of Source T (black dots). The red solid and dashed lines show the best-fitting power-law spectrum and its $1 \sigma$ statistic error band. Shaded gray regions (right axis) show the TS values of the four energy bins. The blue dots are the HESS observations of HESS J1731-347 in the VHE band (Abramowski et al. 2011).

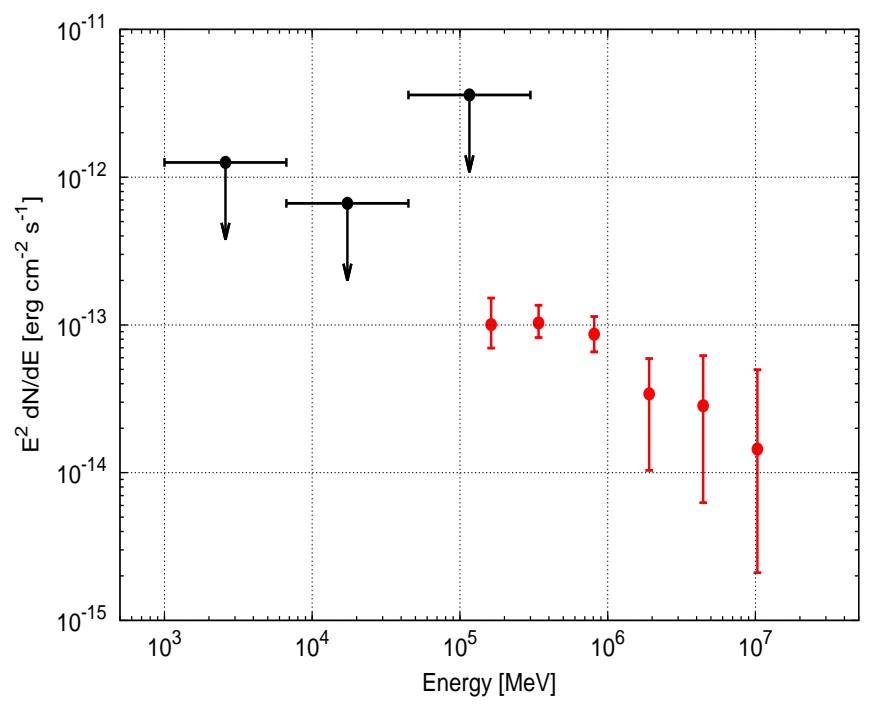

FIG. 4.- Upper limits of $\mathrm{GeV} \gamma$-ray emission from the direction of HESS J1729-345, together with the HESS observations in the VHE band (Abramowski et al. 2011).

to be an exponential cutoff power-law form

$$
d N / d E \propto E^{-\alpha_{i}} \exp \left[-\left(E / E_{c, i}\right)^{\delta}\right],
$$

where $i=e$ or $p, \alpha_{i}$ is the spectral index, $E_{c, i}$ is the cutoff energy of particles. and $\beta$ describes the sharpness of the cutoff. $\delta$ describes the sharpness of the cutoff, and we adopt the typical values of $0.5,0.6$ and 1.0 to constrain the parameters in the model. The radius of the SNR is nearly $0.25^{\circ}$ in the radio band (Tian et al. 2008; Nayana et al. 2017), and $0.27^{\circ}$ in the TeV band (Abramowski et al. 2011). Such an angular size corresponds to a physical radius of about $14-15$ pc for a distance of $3.2 \mathrm{kpc}$. The gas density in the vicinity of HESS J1731-347 is quite uncertain, due to the lack of thermal X-ray emissions. We assume a nominal value of $n=1.0 \mathrm{~cm}^{-3}$.

For the leptonic model, the background radiation field considered includes the cosmic microwave background
(CMB), and an infrared (IR) radiation field with a temperature of $40 \mathrm{~K}$ and an energy density of $1 \mathrm{eV} \mathrm{cm}^{-3}$ (Abramowski et al. 2011). The magnetic field strength is taken as a free parameter, which is determined through fitting to the multi-wavelength data. The derived model parameters are given in Table 3. The corresponding multi-wavelength SED of the model calculation is shown in the left panel of Figure 5.

The leptonic models with the three different values of $\delta$ can reproduce the muti-wavelength SED with little differences. Compared with the results of Yang et al. (2014), the spectral index of electrons $\alpha_{e}$ and cutoff energy $E_{c, e}$ are both slightly smaller in this work. This may due to the updated radio, $\mathrm{X}$-ray and $\mathrm{GeV}$ data we used in the model. The magnetic field strength, $B \sim 28 \mu \mathrm{G}$, is consistent with that given in Yang et al. (2014). Such a magnetic field strength is slightly larger than that of several other SNRs which show similar GeV-TeV $\gamma$-ray spectra, e.g. RX J1713.7-3946 (Abdo et al. 2011; Yuan et al. 2011; Zeng et al 2017), RX J0852-4622 (Vela Junior; Tanaka et al. 2011), and RCW 86 (Yuan et al. 2014). These SNRs are believed to be a class of sources with leptonic origin of the $\gamma$-ray emission (Yuan et al. 2012; Funk 2015; Guo et al. 2017).

The cutoff of the spectrum may be due to the (synchrotron) cooling of electrons. The synchrotron cooling time scale of HESS J1731-347 is estimated to be

$$
t_{\mathrm{syn}} \approx 1800\left(\frac{E_{c, e}}{9 \mathrm{TeV}}\right)^{-1}\left(\frac{B}{28 \mu \mathrm{G}}\right)^{-2} \mathrm{yr} .
$$

This time scale is close to the minimum value of the age of HESS J1731-347 inferred with other methods (Tian et al. 2008; Abramowski et al. 2011; Fukuda et al. 2014; Acero et al. 2015b).

Nayana et al. (2017) reported an anti-correlation between the $\mathrm{TeV} \gamma$-ray emission and radio brightness profile, and ascribed such an anti-correlation to the synchrotron cooling effect with a non-uniform magnetic field. This result supports the leptonic scenario for the multi-wavelength emission of HESS J1731-347.

The right panel of Figure 5 shows the multi-wavelength SED of the hadronic-leptonic hybrid model, in which the radio to X-ray data is acounted for by the synchrotron emission of electrons, and the GeV-TeV $\gamma$-ray emission is produced by the decay of neutral pions from $p p$ collisions. The model parameters are also summarized in Table 3. For the hybrid models with different values of $\delta$, a hard spectral index of protons with $\alpha_{p} \sim 1.7$ even $\alpha_{p} \sim 1.5$, is needed to explain the hard $\mathrm{GeV} \gamma$-ray spectrum. However, such spectrum of protons is difficult to be produced in the conventional diffusive shock acceleration model of strong shocks. The total energy of protons above $1 \mathrm{GeV}$ is estimated to be $W_{p} \sim 1.5 \times 10^{50}\left(n / 1.0 \mathrm{~cm}^{-3}\right)^{-1}(d / 3.2 \mathrm{kpc})^{2}$ erg, corresponding to $\sim 15 \%$ particle acceleration efficiency for a typical total energy of $E_{\mathrm{SN}} \sim 10^{51}$ erg released by a core-collapse supernova. The total energy $W_{p}$ depends on the distance and ambient gas density of HESS J1731-347. Since there is no thermal X-ray emission observed, the gas density would be very low, (e.g., Abramowski et al. (2011) derived an upper limit of gas density of $\sim 0.01 \mathrm{~cm}^{-3}$ assuming an electron plasma temperature of $1 \mathrm{keV}$ ), and hence the corresponding $W_{p}$ would be much higher. However, if HESS J1731-347 expands in an inhomogeneous environment with dense gas clumps, the hard $\gamma$-ray emission and the high energy budget can be solved 
(Inoue et al. 2012; Gabici \& Aharonian 2014; Fukui 2013).

A spatial correlation between the $\mathrm{TeV} \gamma$-ray shell and the interstellar protons at a distance of $\sim 5.2 \mathrm{kpc}$ was reported in Fukuda et al. (2014). It was suggested that the hadronic process contributes a large fraction of the $\gamma$-ray emission of HESS J1731-347 (Fukuda et al. 2014). This is similar to the cases of RX J1713.7-3946 and RX J0852.04622 (Fukui et al. 2012; Fukui 2013). However, no significant emission from dense molecular gas at such a distance was detected by Maxted et al. (2017), which seems to be challenge to the hadronic scenario.

HESS J1729-345 is an unidentified TeV source near HESS J1731-347 (Abramowski et al. 2011). Assuming that HESS J1731-347 locates at a distance of $\sim 3.2 \mathrm{kpc}$, Cui et al. (2016) suggested that the TeV $\gamma$-ray emission of HESS J1729345 possibly originates from the nearby molecular clouds illuminated by the CRs escaped from HESS J1731-347. Capasso et al. (2016) reported a good spatial coincidence between the $\mathrm{TeV} \gamma$-ray image in the bridge region and the dense gas at a distance of $3.2 \mathrm{kpc}$, which further supports the scenario of Cui et al. (2016). Nayana et al. (2017) detected possible radio counterparts of HESS J1729-345 at $843 \mathrm{MHz}$ and $1.4 \mathrm{GHz}$. However, the multi-wavelength data of HESS J1729-345 is still lack. Future multi-wavelength observations are need to explore its nature.

\section{CONCLUSION}

In this paper, we report the $\mathrm{GeV} \gamma$-ray emission from the direction of HESS J1731-347 at a significance level of $\sim 4.7 \sigma$, with nine years of Pass 8 data recorded by the FermiLAT. The spatial morphology of HESS J1731-347 is found to be slightly extended in the GeV band. The GeV spectrum can be described by a hard PL for with an index of $\Gamma=1.77 \pm 0.14$.

The $\gamma$-ray characteristics of HESS J1731-347 is similar with several shell-type SNRs, including RX J1713.7-3946, RX J0852-4622, RCW 86, and SN 1006. A pure leptonic model can account for the wide-band SED of HESS J1731347. If the hadronic process is adopted to explain the $\gamma$-ray emission, a very hard $(\sim 1.6)$ proton spectrum is required. In addition, the energy budget of CR protons may be a problem, given a potentially low gas density environment implied by the lack of thermal X-ray emission.

We also search for $\mathrm{GeV} \gamma$-ray emission from the nearby source HESS J1729-345. No significant excess is detected in its direction, and the upper limits are given. More multiwavelength observations are necessary to address its emission mechanism, and test the proposed scenario of the interaction between CRs escaped from HESS J1731-347 and the molecular clouds.

\section{ACKNOWLEDGMENTS}

We thank V. Doroshenko for providing the new X-ray data. This work is supported by National Key Program for Research and Development (2016YFA0400200), the National Natural Science Foundation of China (Nos. 11433009, 11525313, 11722328, 11703093), Natural Science Foundation of Jiangsu Province of China (No. BK20141444), and the 100 Talents program of Chinese Academy of Sciences.

\section{REFERENCES}

Abdo, A. A., Ackermann, M., Ajello, M., et al. 2010a, ApJL, 710, L92

Abdo, A. A., Ackermann, M., Ajello, M., et al. 2010b, Sci, 327, 1103

Abdo, A. A., Ackermann, M., Ajello, M., et al. 2011, ApJ, 734, 28

Abramowski, A., Acero, F., Aharonian, F., et al. 2011, A\&A, 531, A81

Abramowski, A., Aharonian, F., Ait Benkhali, F. et al. arXiv:1601.04461

Acciari, V. A., Aliu, E., Arlen, T., et al. 2009, ApJL, 698, L133

Acero, F., Ackermann, M., Ajello, M., et al. 2015, ApJS, 218, 23

Acero, F., Aharonian, F., Akhperjanian, A. G., et al. 2010, A\&A, 516, A62

Acero, F., Lemoine-Goumard, M., Renaud, M., et al. 2015, A\&A, 580, A74

Ackermann, M., Ajello, M., Allafort, A., et al. 2013, Sci, 339, 807

Aharonian, F., Akhperjanian, A. G., Aye, K.-M., et al. 2004, Natur, 432, 75

Aharonian, F., Akhperjanian, A. G., Bazer-Bachi, A. R., et al. 2005, A\&A, 437, L7

Aharonian, F., Akhperjanian, A. G., Bazer-Bachi, A. R., et al. 2006, A\&A, 449,223

Aharonian, F., Akhperjanian, A. G., Bazer-Bachi, A. R., et al. 2007a, A\&A, 464, 235

Aharonian, F., Akhperjanian, A. G., Bazer-Bachi, A. R., et al. 2007b, ApJ, 661,236

Aharonian, F., Akhperjanian, A. G., de Alemida, U. B., et al. 2008a, A\&A, 486,829

Aharonian, F., Akhperjanian, A. G., de Almeida, U. B., et al. 2008b, A\&A, 477,353

Aharonian, F., Akhperjanian, A. G., de Almeida, U. B., et al. 2009, ApJ, 692,1500

Albert, J., Aliu, E., Anderhub, H., et al. 2007a, A\&A, 474, 937

Albert, J., Aliu, E., Anderhub, H., et al. 2007b, ApJL, 664, L87

Araya, M, 2017, ApJ, 843, 12

Atwood, W. B., Abdo, A. A., Ackermann, M., et al. 2009, ApJ, 697, 1071

Bamba, A., Pühlhofer, G., Acero, F., et al. 2012, ApJ, 756, 149

Capasso, M., Condon, B., Coffaro, M., et al. 2016, arXiv:1612.00258

Condon, B., Lemoine-Goumard, M., Acero, F. \& Katagiri, H. 2017, arXiv: 1711.05499

Cui, Y., Pühlhofer, G., \& Santangelo, A. 2016, A\&A, 591, A68

Doroshenko, V., Pühlhofer, G., Bamba, A., et al. 2017, arXiv:1708.04110
Fukuda, T., Yoshiike, S., Sano, H., et al. 2014, ApJ, 788, 94

Fukui, Y., Sano, H., Sato, J., et al. 2012, ApJ, 746, 82

Fukui, Y. 2013, in Astrophysics and Space Science Proc., Vol. 34, Cosmic

Rays in Star-Forming Environments, ed. D. F. Torres \& O. Reimer

(Berlin: Springer), 249

Funk, S. 2015, Annual Review of Nuclear and Particle Science, 65, 245

Gottschall, D., Capasso, M., Deil, C., et al. 2016, arXiv:1612.00261

Gabici, S. \& Aharonian, F. A. 2014, MNRAS, 445, L70

Guo, X.-L., Xin, Y.-L., Liao, N.-H., et al. 2017, ApJ, 835, 42

Halpern, J. P., \& Gotthelf, E. V. 2010, ApJ, 710, 941

Hewitt, J. W., Grondin, M.-H., Lemoine-Goumard, M., et al. 2012, ApJ, 759,89

Inoue, T., Yamazaki, R., Inutsuka, S., \& Fukui, Y. 2012, ApJ, 744, 71

Koyama, K., Petre, R., Gotthelf, E. V., et al. 1995, Nature, 378, 255

Li, H. \& Chen, Y. 2010, MNRAS, 409, L35

Li, H. \& Chen, Y. 2012, MNRAS, 421, 935

Maxted, N., Burton, M., Braiding, C., et al. 2017, arXiv:1710.06101

Nayana, A. J., Chandra, P., Roy, S., et al. 2017, MNRAS, 467, 155

Rieger, F. M., de Oña-Wilhelmi, E., \& Aharonian, F. A., 2013, Front. Phys., 8,714

Tanaka, T., Allafort, A., Ballet, J., et al. 2011, ApJL, 740, L51

Tian, W.-W., Leahy, D. A., Haverkorn, M., \& Jiang, B. 2008, ApJL, 679, L85

Tian, W.-W., Li, Z., Leahy, D. A., et al. 2010, ApJ, 712, 790

Xin, Y.-L., Liang, Y.-F., Li, X., et al. 2016, ApJ, 817, 64

Xin, Y.-L., Guo, X.-L., Liao, N.-H., et al. 2017, ApJ, 843, 90

Xing, Y., Wang, Z., Zhang, X., \& Chen, Y. 2016, ApJ, 823, 44

Yang, R.-Z., Zhang, X., Yuan, Q., \& Liu, S.-M. 2014, A\&A, 567, A23

Yuan, Q., Huang, X.-Y., Liu, S.-M., \& Zhang, B. 2014, ApJL, 785, L22

Yuan, Q., Liu, S.-M., \& Bi, X.-J. 2012, ApJ, 761, 133

Yuan, Q., Liu, S. M., Fan, Z. H., Bi, X. J., \& Fryer, C. L. 2011, ApJ, 735, 120

Zeng, H.-D., Xin, Y.-L., Liu, S.-M., et al. 2017, ApJ, 834, 153 

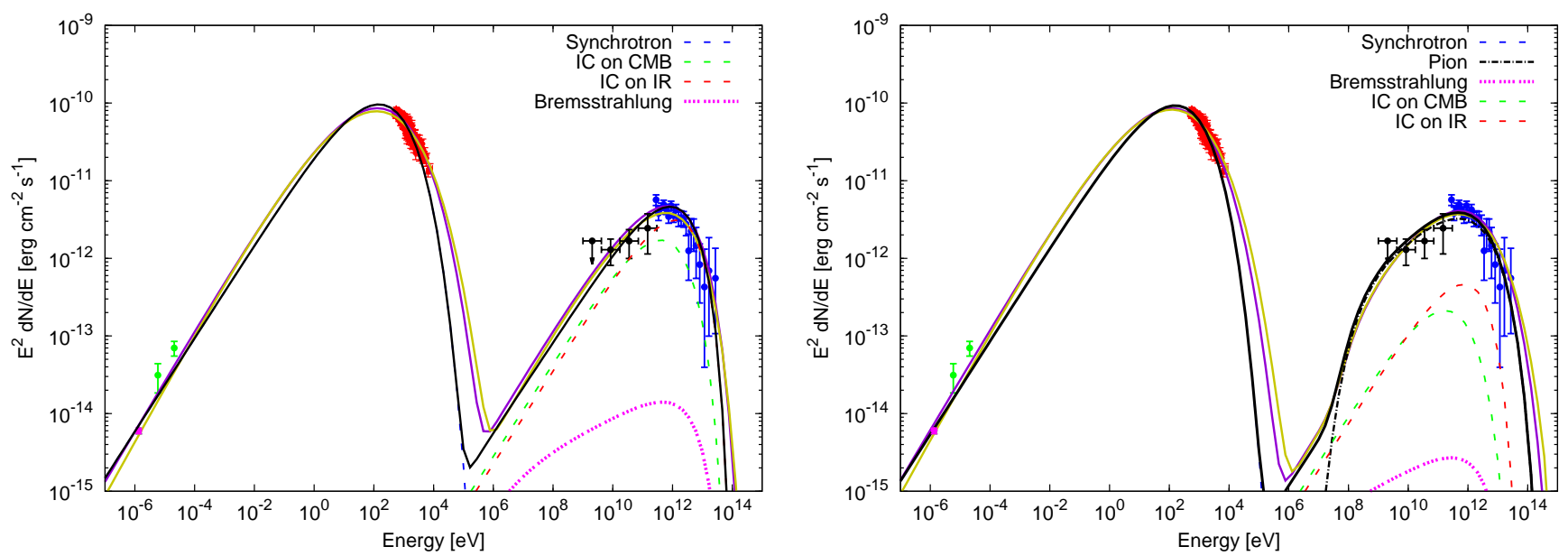

FIG. 5.- Modeling of the multi-wavelength SED of HESS J1731-347. The left panel is for the leptonic model, and the right panel is for the hadronic-leptonic hybrid model. The models with $\delta=0.5,0.6$, and 1.0 are presented by the dark-yellow, purple and black solid lines, respectively. The dashed and dotted lines with different colors represent the different radiation components for the model of $\delta=1.0$. The observational data of the radio (magenta for Nayana et al. (2017); green for Tian et al. (2008)), X-rays (Doroshenko et al. 2017), TeV $\gamma$-rays (Abramowski et al. 2011), and GeV $\gamma$-rays presented in this work are shown.

TABLE 3

MODEL PARAMETERS

\begin{tabular}{cccccccccc}
\hline \hline Model & $\delta$ & $\alpha_{p}$ & $\alpha_{e}$ & $\begin{array}{c}B_{\mathrm{SNR}} \\
(\mu \mathrm{G})\end{array}$ & $\begin{array}{c}W_{e} \\
\left(10^{47} \mathrm{erg}\right)\end{array}$ & $\begin{array}{c}E_{c, e} \\
(\mathrm{TeV})\end{array}$ & $\begin{array}{c}W_{p} \\
\left(10^{50} \mathrm{erg}\right)\end{array}$ & $\begin{array}{c}E_{c, p} \\
(\mathrm{TeV})\end{array}$ & $\chi^{2}$ \\
\hline Leptonic & 0.5 & - & 1.6 & 29.0 & 1.7 & 1.2 & - & - & $529.27 / 320$ \\
& 0.6 & - & 1.7 & 27.0 & 2.0 & 2.8 & - & - & $428.96 / 320$ \\
& 1.0 & - & 1.8 & 28.0 & 1.6 & 8.9 & - & - & $406.27 / 320$ \\
\hline Hybrid & 0.5 & 1.5 & 1.6 & 85.0 & 0.35 & 0.7 & 1.4 & 10.0 & $472.04 / 317$ \\
& 0.6 & 1.5 & 1.7 & 87.0 & 0.35 & 1.5 & 1.5 & 15.0 & $372.33 / 317$ \\
& 1.0 & 1.7 & 1.8 & 80.0 & 0.3 & 5.5 & 1.5 & 38.0 & $377.31 / 317$ \\
\hline \hline
\end{tabular}

Note. - The total energy of relativistic particles, $W_{e, p}$, is calculated for $E>1 \mathrm{GeV}$. 